\title{
Educación contable, cuarentena y medios digitales Retos, oportunidades, tensiones y experiencias desde Colombia
}

Mary A.Vera-Colina (maaveraco@unal.edu.co)
Zuray Melgarejo (zamelgarejomo@unal.edu.co)
Claudia Niño Galeano (clninog@unal.edu.co)

Se presenta un análisis interpretativo-narrativo

Profesoras - Universidad Nacional de Colombia - Grupo de Estudios de las experiencias reportadas por profesores y auxiliares de docencia de cursos contables en la Universidad Nacional de Colombia, con el propósito de comprender los retos, oportunidades y tensiones que se observan durante los primeros meses de la pandemia COVID-19, en un contexto de cambio a docencia online. Los resultados muestran un comportamiento compatible con las etapas del duelo de Bowlby (1980) que avanzan hacia una reorganización de sus comportamientos. Se espera que la investigación contribuya a la identificación de prácticas académicas que faciliten el enfrentamiento de imprevistos que demanden cambios inmediatos desde esfuerzos individuales e institucionales.

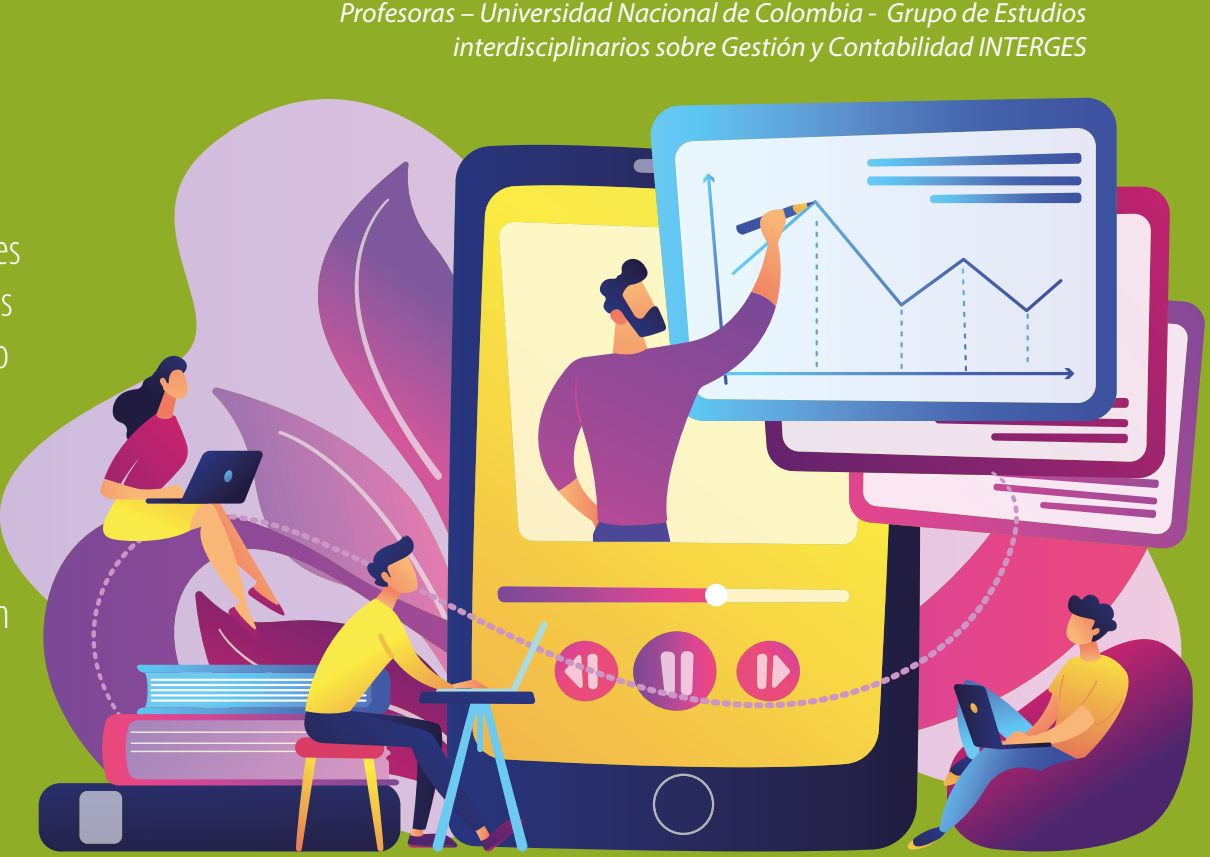

\section{Introducción y contexto}

a declaración internacional de pandemia de COVID-19, y la presencia de los primeros casos de contagio en Colombia, llevaron al cierre indefinido de colegios y universidades desde el 16 de marzo de 2020. Esta decisión fue ratificada por normativas del gobierno nacional, de gobernaciones y alcaldías que ordenaron acciones de cuarentena y aislamiento obligatorio en actividades consideradas con presencialidad no esencial, entre ellas la educación en sus diferentes niveles.

Como caso de estudio particular, exponemos algunas experiencias recopiladas dentro del programa de Contaduría Pública de la Universidad Nacional de Colombia (UNC) con sede en Bogotá, en el cual el semestre regular de clases presenciales en el campus estaba pro- gramado del 03 de marzo al 25 de junio, sin haber previsto la situación de contingencia que traería la pandemia que obligaría a modificar abruptamente la metodología educativa y a implementar estrategias de clases remotas mediadas por recursos tecnológicos y conexión a internet.

La UNC es la universidad pública más reconocida en el país, con una presencia significativa en América Latina. Su programa de Contaduría Pública se encuentra acreditado con alta calidad y se destaca entre los primeros lugares de la región, por lo que resulta relevante comprender cómo institucionalmente ha replanteado su estrategia de formación en el escenario que impone la pandemia.

El análisis de la reacción institucional requiere conocer las actuaciones de sus actores principales: profesores y estudiantes. En este con- texto, surgen preguntas relacionadas sobre las reacciones que ellos han tenido ante estos cambios inesperados, y cómo sus prácticas docentes y de aprendizaje han debido replantearse para enfrentar el nuevo escenario educativo: ¿cuáles fueron sus reacciones iniciales? ¿cómo modificaron sus actividades? ¿cómo perciben su desempeño en este contexto? ¿se identifican retos, oportunidades y tensiones similares en diferentes experiencias?

Como una primera aproximación a estas reflexiones, este documento persigue como objetivo interpretar las narrativas de profesores y estudiantes auxiliares que lideran cursos de contabilidad financiera en la UNC, como parte de los grupos de interés a destacar en este complejo panorama, destacando los retos, oportunidades, tensiones que se evidencian en sus experiencias durante los primeros meses de pandemia. 


\section{Metodología y datos}

Inicialmente, las reflexiones docentes han sido recopiladas a través de relatos (escritos) solicitados a un grupo de 2 profesoras y sus 3 estudiantes auxiliares de docencia (monitores). Estas reflexiones están centradas en sus reacciones iniciales al anuncio de pandemia y cambio de metodología educativa, las acciones implementadas durante el semestre y sus reflexiones al finalizar este primer período del año, destacando los retos, oportunidades y tensiones que pudieron describir en sus textos. Las respuestas se reciben de manera voluntaria e independiente durante el mes de septiembre de 2020 y no hay relatos cruzados entre ellos, por lo cual sus escritos se refieren a estudiantes y profesores de manera generalizada y no solamente a los 5 participantes en esta fase de la investigación. Se aplica la metodología de análisis cualitativo-interpretativo de narrativas (Creswell, 2013; Beattie, 2014; Dornelles y Sauerbronn, 2019), que permite la identificación de sus experiencias durante el período semestral (marzo a julio de 2020, ante la ampliación de fechas aprobada por las directivas universitarias). La interpretación se realiza de manera agrupada y anónima para procurar la confidencialidad de los participantes.

En este documento, la interpretación de resultados se centra en estos escritos, como una primera aproximación a algunas experiencias que pueden resultar comunes en Colombia y otras naciones. Se proyecta ampliar estos resultados en fases futuras de la investigación, incluyendo otros perfiles docentes y estudiantiles en la recopilación de los relatos.

La figura 1 muestra las palabras utilizadas con mayor frecuencia en las narrativas.

\section{Reacciones iniciales}

El anuncio de cierre del campus universitario fue publicado el domingo 15 de marzo de 2020. Profesoras y estudiantes nos comparten sus sensaciones previas de zozobra y temor ante los riesgos de contagio, ahora aumentadas al confirmar la gravedad de la situación con la decisión de las directivas universitarias. Además de las angustias personales y familiares, los relatos expresan diferentes percepciones, tales como:

- Desconcierto e incertidumbre ante la continuidad de las clases en modalidad virtual, dado que los cursos no fueron diseñados bajo esa metodología. Dos de las narrativas describen que se consideró necesario hacer una pausa en las clases de esa primera semana de cuarentena para poder asimilar la situación y

Figura 1

Nube de palabras extraída de los relatos narrativos sobre experiencias educativas durante la pandemia COVID-19

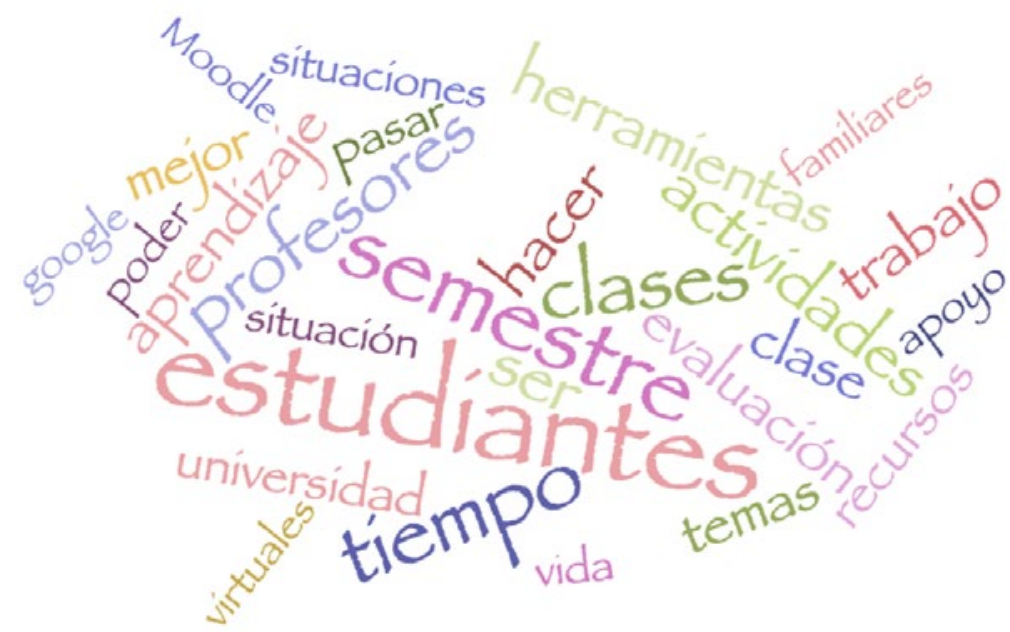

Fuente: elaboración propia con base en los relatos narrativos

planificar los primeros cambios a implementar. Todos coinciden en la necesidad de replantear sus estrategias de docencia y aprendizaje con la mejor planificación posible en el nuevo contexto.

- La profunda desigualdad socio-económica del país agudizó el problema de acceso a la educación de calidad en este período, ya que un sector importante de la población no contaba con la conexión adecuada a Internet ni disponía de los equipos tecnológicos adecuados para el cambio a la metodología de clases remotas. La mayor parte de los estudiantes de la UNC provienen de familias de bajos ingresos y dependen de los recursos tecnológicos disponibles en el campus, ahora cerrado indefinidamente.

- Profesoras y estudiantes comparten la preocupación que se generó sobre las condiciones de estudio-trabajo en el hogar, en espacios que no fueron diseñados para esta realidad. Adicionalmente, se vio afectado el balance trabajo-estudio-vida personal, ya que deben asumirse nuevas responsabilidades no sólo en lo académico sino también en los asuntos familiares.

- Las profesoras tomaron la iniciativa de aplicar encuestas a sus estudiantes para comprender mejor el contexto, limitaciones, preocupaciones, recursos disponibles, y con esta información planificar actividades pertinentes y viables para la mayoría de ellos.

- Para la continuidad del proceso de aprendizaje, una de las preocupaciones principales fue la modificación de las metodologías de evaluación. Uno de los relatos de estudiantes señala que "... pareció interesante que muchos parciales que iban a ser de memoria, pasaron a ser de reflexión, de crítica y de entender los conceptos, lo cual es muy beneficioso para una futura vida laboral".

- A pesar de las condiciones adversas, los relatos muestran la mejor disposición para asumir con optimismo y compromiso la nueva realidad, buscando implementar acciones que permitan continuar los procesos de aprendizaje. Entre los aspectos positivos se destaca el ahorro en el tiempo de traslado desde los hogares a la universidad, así como el fortalecimiento del trabajo en equipo basado en herramientas virtuales.

\section{Acciones implementadas y desarrollo del semestre con clases remotas}

Luego de superar las reacciones iniciales, los narradores nos comparten las situaciones experimentadas durante el semestre:

- Un obstáculo para el proceso de aprendizaje con base en medios digitales fue la timidez de muchos estudiantes y bajos niveles de interacción en las clases (profesor-estudiantes y estudiantes-estudiantes). Esta situación fue mejorando paulatinamente pero fue un aspecto considerado como relevante para el logro de los objetivos de cada curso.

- También se destaca la sobrecarga de actividades para profesores y estudiantes, llegando a describirla como abrumadora. Un relato estudiantil lo describe así: "... el profesor se me había convertido en un monstruo que dejaba un montón de archivos pdf por leer y una cantidad de talleres (a veces absurdos), y que esperaban 


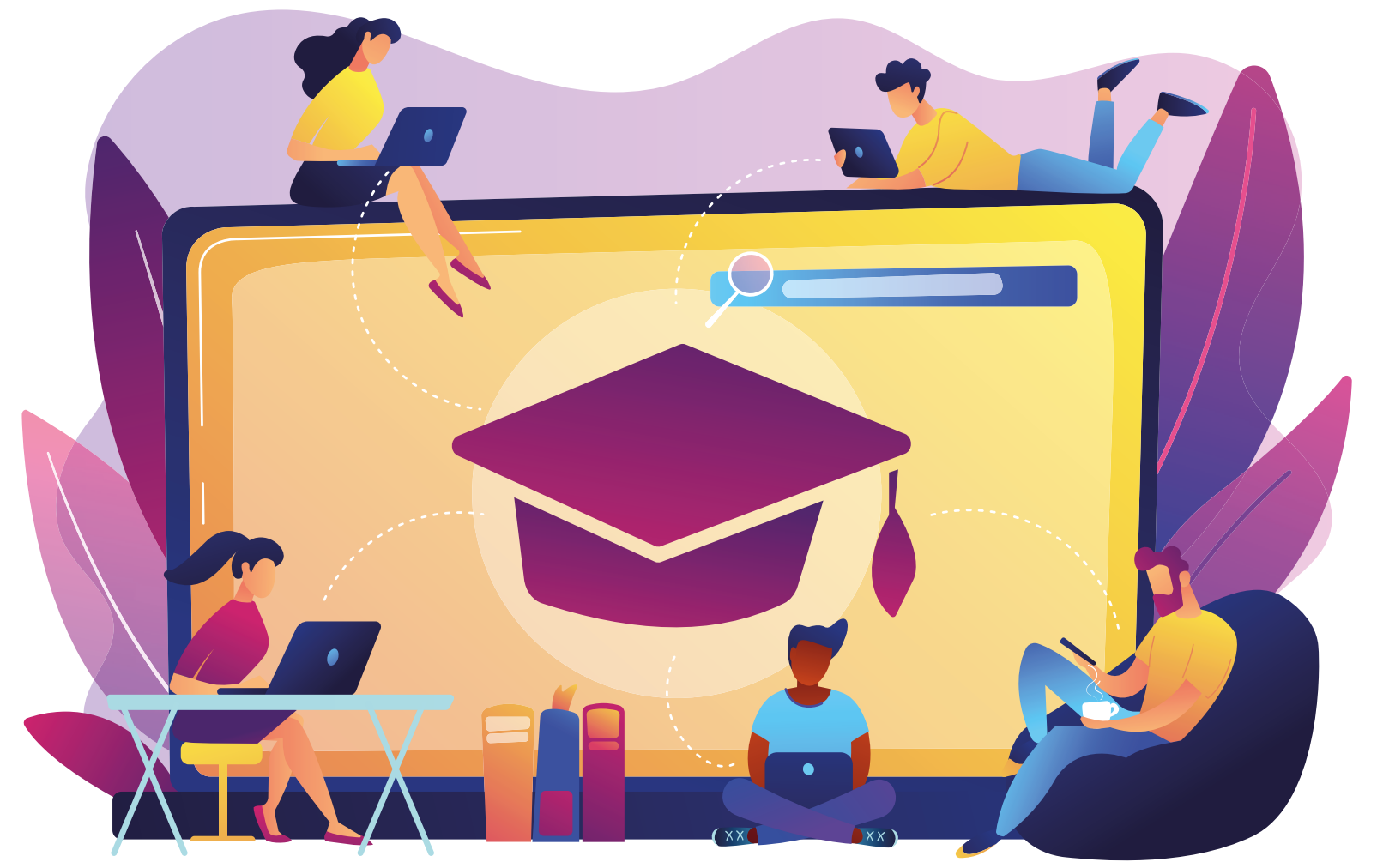

a la fecha del parcial para mirar si aprendimoso no por nuestra cuenta, donde el estudiante era el malo por no aprender...." Este agotamiento es un factor común en las narrativas.

- Se reconoce el esfuerzo de los profesores que promovieron el aprendizaje autónomo en el marco de los limitados recursos disponibles de los estudiantes y dificultades en la conexión a Internet. Se implementaron actividades asincrónicas y grabación de video clases que permitían avanzar en el desarrollo de los cursos con ritmos diferentes.

- Las estrategias de evaluación descritas en los relatos de las profesoras privilegiaron el aprendizaje basado en problemas y promoción de pensamiento crítico. También se consideraron como valiosas las actividades lúdicas, el trabajo en equipo y metodologías activas, más allá de la docencia tradicional de clase magistral.

- Un comentario interesante es la valoración del apoyo de la institución a sus docentes a través de algunas capacitaciones para mejorar el uso de herramientas digitales, al mismo tiempo que los estudiantes auxiliares (monitores) manifiestan como desfavorable no haber sido incluidos en estas actividades a pesar de su rol de apoyo en las clases.

- Los relatos coinciden al destacar las permanentes dificultades para equilibrar el tiempo dedicado a estudio-trabajo-vida personal, y a compartir el espacio hogareño con las actividades académicas. Este desbalance dificulta

\section{"Como una primera aproximación a estas reflexiones, este} documento persigue como objetivo interpretar las narrativas de profesores y estudiantes auxiliares que lideran cursos de contabilidad financiera en la UNC, como parte de los grupos de interés a destacar en este complejo panorama, destacando los retos, oportunidades, tensiones que se evidencian en sus
experiencias durante los primeros meses de pandemia"

la concentración, profundiza la sensación de agotamiento y se reconoce la necesidad de mejorar este equilibrio en los meses futuros.

\section{Reflexiones al final del semestre académico y sobre cambios futuros}

Alivio y alegría son las palabras más utilizadas para referirse a la finalización del primer período semestral, aunque persisten las preocupaciones sobre los retos que trae la segunda mitad de año con la continuidad de las clases remotas. Las narraciones reiteran en más de una oportunidad esta sensación de satisfacción, reflexionando sobre los resultados alcanzados en un contexto marcado por los imprevistos y las múltiples dificultades. Se plantea que “... se superó el caos de la mejor manera posible...", de manera sobresaliente y con muchas mejoras a implementar en el siguiente período. Algunos aspectos a destacar en las narraciones:
- Las profesoras perciben su trabajo como un esfuerzo aislado o individual, que puede potenciarse con mayor cooperación y apoyo institucional.

- La evaluación de desempeño registrada por los estudiantes sobre el trabajo de las profesoras que participaron en la construcción de las narrativas resultó altamente favorable, destacando una adecuada adaptación al nuevo contexto y apoyo permanente a sus necesidades de aprendizaje.

- Se requiere fortalecer la capacitación y aprendizaje para mejorar el uso de los medios digitales herramientas, tanto en estudiantes como profesores, con mayor apoyo institucional. Es importante promover el trabajo en equipo de los docentes en el uso de las tecnologías y diseño de recursos instruccionales.

- Una tensión que persiste a lo largo del pe- 
ríodo es el desbalance de tiempo dedicado mayormente a actividades académicas descuidando aspectos de la vida personal (familia, pareja, hijos, ejercicio físico, salud, ocio, descanso). Todos consideran que es un aspecto al que debe prestarse la debida atención para evitar mayores conflictos emocionales y aliviar el agotamiento que describen los participantes.

- Se reitera la reflexión sobre los retos a ser enfrentados en un nuevo semestre, relacionados con "...mejorar las habilidades para diseñar actividades y dar clases a través de diferentes plataformas digitales y herramientas educativas... Ser aún más consciente que son distintas las características socioeconómicas, las condiciones materiales, las habilidades y capacidades de aprendizaje de cada estudiante".

\section{Consideraciones finales}

Al interpretar las narrativas recopiladas se evidencia un comportamiento compatible con la teoría del apego (attachment theory) y las cuatro fases del duelo propuestas por Bowlby (1980: 85): aturdimiento-angustia, nostalgia y búsqueda de la figura perdida (que origina el duelo), desorganización y desesperación, reorganización. Son fases asociadas al duelo por la pérdida de un ser querido que pueden usarse como analogía para comprender mejor las experiencias que describen los narradores en su paso por la crisis COVID-19, aún sin solución a la vista. El autor explica que las fases no tienen una duración definida y cada persona puede estar circulando entre dos fases de manera repetitiva, pero son cuatro momentos que describen muy bien los episodios de un duelo.

En los relatos se perciben sentimientos encontrados ante lo imprevisto, con una reacción optimista que facilita el cambio y la adaptación (reorganización). No se puede afirmar que se alcanza una fase de "aceptación" como lo sugieren Kubler-Ross \& Kessler (2005), pero si se observa un proceso que conduce a adaptar (reorganizar) el compor- tamiento académico al nuevo contexto que continuará dominando la realidad mundial por tiempo indefinido.

También se observan algunas similitudes con los hallazgos de Sangster, Stoner \& Flood (2020), en cuanto a las preocupaciones sobre las modificaciones a realizar en las metodologías de evaluación, el deseo de reducir el estrés y mejorar el balance trabajo-estudio-vida personal.

Como principales retos a afrontar para lograr un proceso de aprendizaje exitoso se mencionan situaciones asociadas a desigualdad socio-económica, dificultades técnicas para la conexión a internet, limitaciones en los recursos tecnológicos disponibles y en habilidades de docentes y estudiantes para aplicar metodologías basadas en medios digitales. Las narrativas describen cómo se ha intentado atender cada reto, especialmente aquellos asociados a la mejora de las habilidades de los participantes por tratarse de una situación en la que tienen mayor control para aplicar soluciones apropiadas. En el caso de la desigualdad de condiciones, situación que muchas veces supera el alcance de las soluciones que pueden ofrecer los profesores y sus auxiliares, los casos más críticos fueron informados al departamento de bienestar universitario para su debida atención institucional.

Por otra parte, se reitera que la crisis también permite explorar oportunidades de mejora para el contexto educativo, entre ellos, la posibilidad de implementar metodologías activas (Nogueira et al, 2020; Araújo et al, 2017) así como de poner en práctica actividades de aprendizaje y evaluación que promuevan el pensamiento crítico, coincidiendo con lo reportado por Sangster, Stoner \& Flood (2020).

Al describir las tensiones y conflictos que generaron mayores preocupaciones en estos primeros meses de la pandemia, es evidente que la gestión del tiempo y el equilibrio entre estudio-trabajo y vida personal-familiar son asuntos importantes aún por resolver, especialmente en el marco de los riesgos asociados a la pandemia y cómo estos pueden llegar a afectar la salud propia y de los seres queridos. Ninguno de los relatos afirma que se hayan presentado situaciones de contagio en los participantes.

En medio de sus tensiones, es importante destacar la sensibilidad que expresan los narradores ante la situación de los estudiantes en condiciones de desigualdad exacerbada, ante lo cual manifiestan empatía y sororidad, no solo en los relatos sino también en las acciones implementadas en sus cursos.

Este documento interpreta los resultados preliminares de una investigación en proceso, donde no se pretende obtener hallazgos estadísticamente significativos sino avanzar en la comprensión de la diversidad de situaciones que los académicos atienden en este complejo contexto. En fases futuras del estudio se espera ampliar el número de participantes y diversidad de perfiles para seguir profundizando en esta interpretación sobre las reacciones de los académicos contables ante la crisis. También se espera ampliar las narrativas a las experiencias relacionadas con los procesos de investigación, no considerados en esta etapa.

Se espera que la investigación contribuya a la identificación de prácticas académicas que faciliten el enfrentamiento de situaciones imprevistas que demanden cambios inmediatos. La interpretación de tales experiencias permitirá generar recomendaciones de posibles acciones a implementar en casos similares, aplicables a docentes, estudiantes y especialmente a la esfera institucional. Como se extrae de los relatos, los problemas van siendo superados mayormente a través de esfuerzos individuales y aislados, por lo que es relevante identificar los mecanismos que permitan potenciarlos desde el apoyo institucional.

Referencias

Araújo, E., Miranda, G. \& Casa Nova, S. (2017) Revolucionando a Sala de Aula. Editora Gen Atlas.

Beattie, Vivien (2014). Accounting narratives and the narrative turn in accounting research. The British Accounting Review, n. 46, 111-134. http://dx.doi.org/10.1016/j. bar.2014.05.001

Bowlby, J. (1980) Attachment and Loss: Sadness and Depression. Volume III. Howarth Press, London.

Creswell, J. (2013). Qualitative inquiry and research design: choosing among five approaches. California: SAGE Publications, Inc.

Dornelles, O. M., y Sauerbronn, F. F. (2019). Narrativas: Definição e Aplicações em Contabilidade. Sociedade, Contabilidade e Gestão. 14 (4). 19-37. DOI: https://doi. org/10.21446/scg_ufrj.v14i4.27082

Kubler-Ross, E, \& Kessler, D. (2005) On Grief and Grieving: Finding the Meaning of Grief Through the Five Stages of Loss. Scribner, New York.

Nogueira, D., Araújo, E., Miranda, G., Casa Nova, S. (2020) Revolucionando a Sala de Aula 2 - Novas Metodologias Ainda Mais Ativas. Editora Gen Atlas.

Sangster, A., Stoner, G., Flood, B. (2020) Insights into accounting education in a COVID-19 world, Accounting Education, 29:5, 431-562, DOI: 10.1080/09639284.2020.1808487 